\title{
FINITE ELEMENT FORMULATION FOR THE SIMULATION OF HOT SHEET METAL FORMING PROCESSES $†$
}

\author{
SOMNATH GHOSH and NOBORU KIKUCHI \\ Dcpartment of Mcchanical Enginecring and Applied Mechanics, The University of Michigan,
} Ann Arbor, MI 48109, U.S.A.

(Communicated by J. T. ODEN)

\begin{abstract}
Simulation of sheet metal forming processes involves large deformation analysis. In this paper, the finite element method has been utilized for a consistent analysis of coupled thermomechanical large deformation of metals. A time dependent elastic-viscoplastic model has been used to represent the flow type behavior of metals at elevated temperatures. The proposed model incorporates initial anisotropy and temperature dependence of metals. A proper choice of objective stress rate, suitable for anisotropic materials has been made. Integration of the rate constitutive equations is carried by a return mapping algorithm. A special Lagrangian-Eulerian three dimensional finite element mesh description has been used to avoid the typical problems encountered in a Lagrangian description. A technique for solving the coupled equations is suggested.
\end{abstract}

\section{INTRODUCTION}

Hot metal forming processes, such as rolling or sheet metal forming, in general involve large inelastic deformation of the workpiece. A coupled thermomechanical analysis of the large deformation problem is necessary to properly simulate such a process. A number of metals have been noted to cxhibit rate (time) dependent inclastic behavior at elevated tempcratures. The viscous flow of metals at a high temperature, enhances the desirability of an appropriate rate dependent elastic-viscoplastic material model for analysis. Researchers like Kobayashi [19], Dawson et al. [20] and Taylor et al. [21] are among many, who have tried to simulate hot metal forming analysis. In this paper, a detailed theoretical treatment for a coupled thermo-mechanical finite element analysis of the sheet metal forming process has been presented.

There exists a considerable body of literature on the analysis of sheet metal forming process. A finite element procedure using the nonlinear membrane theory of shells has been developed by Wang and Budiansky [22] for rate insensitive materials and has been extended to include rate effects, by Wang and Wenner [23]. Most of these analyses use the membrane theory of shells and are applicable only in cases where bending is insignificant. The assumptions of shell theory restricts the applicability of these models to cases of small strain only. In this paper, we have considered a general three dimensional finite element model for the large deformation problem.

Due to the magnitude of deformation and the nature of contact boundary condition, the conventional Lagrangian mesh poses difficulties of excessive element distortion and unmatched boundaries. These difficulties therefore require remeshing and rezoning of the Lagrangian mesh. In an attempt to circumvent these limitations, a special Lagrangian-Eulerian mesh, whose motion is not restricted by the movement of the material, has been used for stress and temperature analysis.

A strain-hardening overstress viscoplastic model has been chosen to represent the material rate dependence. It is based on a unified viscoplastic model proposed by Perzyna [8] in 1966 which has been modified to include large deformation, thermal effects and initial anisotropy of the prerolled sheet. A static yield criterion has been developed to incorporate the initial anisotropy of the material.

A proper integration algorithm for the rate constitutive equations is essential in large deformation analysis because it has to satisfy the conditions of numerical stability, consistency

† This research was supported by the ONR through grant No. $0001485 \mathrm{~K} 0799$ 
with the constitutive equations and incremental objectivity. A return mapping scheme in conjunction with operator split which satisfies the desired requirements, has been implemented to integrate the rate type constitutive equations.

Finally, a global analysis technique has been suggested for coupling the various separate algorithms, to be used in the finite element simulation. The analysis is done in discrete time steps and within each time step, several different iterations are required, for convergence to the incremental solutions. The quasi-Newton iterative schemes have been found to be most suitable for the types of equations that evolve in this analysis. Numerical results demonstrating the strength of the proposed finite element model, however, have not been discussed here and will be presented in a forthcoming paper.

\section{FINITE ELEMENT FORMULATION USING AN ARBITRARY LAGRANGIAN-EULERIAN DESCRIPTION}

The arbitrary Lagrangian-Eulerian description introduces a reference system which is not constrained to remain fixed in space (Eulerian) or to move with material points in the body (Lagrangian). In this description, the motion of the mesh is represented by a set of arbitrary independent variables which may also represent pure Eulerian or Lagrangian descriptions as limiting cases. The flexibility in the choice of the motion of the reference system exploits the advantages of both the descriptions mentioned above.

An arbitrary Lagrangian-Eulerian mesh was introduced in the context of finite difference solution of Navier-Stokes equation in fluid mechanics by Hirt et al. [1]. Subsequently, it has been used in the finite element modelling of fluid-structure interaction problems by Donea $e t$ al. [2] and Belytschko et al. [3], in viscous flow problems by Hughes et al. [4] and in large deformation of solids by Haber [5]. Haber's description differs from the rest in the fact that, while most of the others have used velocity as their primary kinematic variable, Haber has used a displacement based ALE method. In his paper, Haber has decomposed the deformation into Lagrangian and Eulerian parts, introducing a mapping of each of these onto a reference configuration. The present formulation, despite its use of displacements as the primary kinematic variable, differs from the above work in the fact that the reference configuration in the present formulation moves in the space of motion of the deformable body itself.

As an alternative to the methods of formulation discussed in [2-4] etc, the Reynolds transport theorem has been appplied to an arbitrarily moving control volume with deformable boundary to obtain the field equations of mechanics with respect to an arbitrarily moving grid point.

\subsection{Continuity, momentum and energy equations with respect to an arbitrarily moving grid point}

In the Lagrangian-Eulerian description, the reference configuration comprises of a set of grid points which may be identified by a set of three independent coordinates $\chi_{1}, \chi_{2}$ and $\chi_{3}$ or simply $\chi_{i}$. With no loss of generality $\chi$ may be used to denote the position of a representative grid point at time $t=0$.

At any time $t$, a set of arbitrary grid points constitute a control volume $\Omega(t)$ in space with a deforming boundary $\Gamma(t)$ as shown in Fig. 1. It is assumed that system of material particles constituting a continuum passes through this control volume. This is possible because of the arbitrariness of the motion of the grid points. The Reynolds transport theorem may then be utilized to yield the three governing equations as shown next.

(a) Continuity: From the principle of conservation of mass of a system of particles passing through the control volume, Reynolds transport theorem yields:

$$
\frac{\mathrm{d}}{\mathrm{d} t}\left(\int_{\Omega(t)} \rho \mathrm{d} \Omega\right)+\int_{\Gamma(t)} \rho(\mathbf{V}-\mathbf{W}) \cdot \mathbf{n} \mathrm{d} \Gamma=0
$$

where $\rho$ is the density, $\mathbf{V}$ is the material velocity, $\mathbf{W}$ is the grid point velocity and $\mathbf{n}$ is the unit outward normal to the boundary of the control volume at time $t$. It is to be noted that all of the above variables are functions of $\mathbf{x}$, the current grid point location and time $t$. 


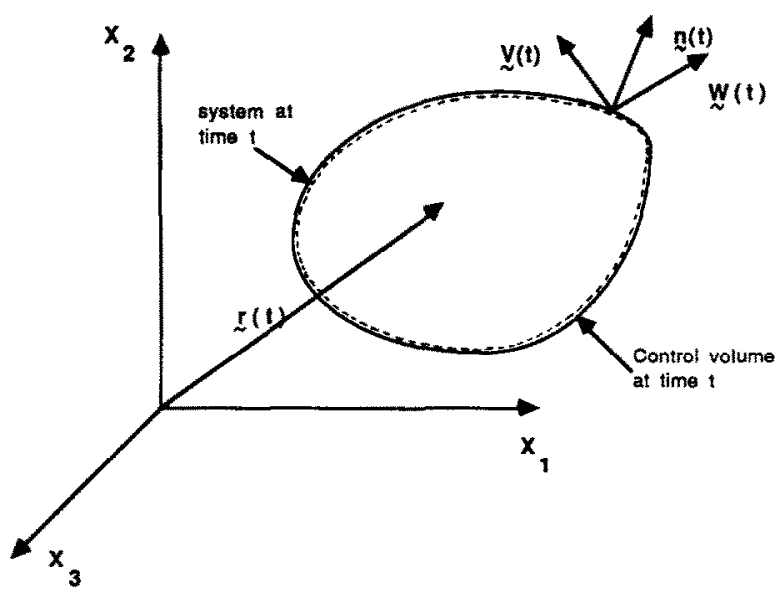

Fig. 1. System of particles coinciding with control volume at time $t$.

In the ALE description, the motion of the grid points may be expressed in terms of the coordinates $\chi_{i}$ by equations of the form

$$
x_{i}=x_{i}\left(\chi_{j}, t\right)
$$

These equations represent a mapping of the initial configuration of grid points on to the current configuration. Although, the motion of the grid points is arbitrary in general, an inverse relation is assumed to exists as:

$$
\chi_{i}=\chi_{i}\left(x_{j}, t\right)
$$

The necessary and sufficient condition for the inverse relation to exist, is that the Jacobian

$$
J=\left|\frac{\partial x_{i}}{\partial x_{j}}\right|
$$

is nonvanishing.

An element of the initial control volume $\Omega_{0}$ may thus be related to a corresponding element of the current configuration $\Omega(t)$ as:

$$
\mathrm{d} \Omega=J \mathrm{~d} \Omega_{0}
$$

Applying the above relation and the divergence theorem to eqn (1), the following equation results.

$$
\int_{\Omega_{0}}\left[\frac{\partial(\rho J)}{\partial t}+J \frac{\partial}{\partial x_{i}}\left(\rho\left(V_{i}-W_{i}\right)\right)\right] \mathrm{d} \Omega_{0}=0
$$

From eqn (6), the fact that the control volume is arbitrary, yields the pointwise continuity equation with respect to a grid point moving independently from the material.

$$
\left.\frac{\partial(\rho J)}{\partial t}\right|_{\chi}+J \frac{\partial}{\partial x_{i}}\left(\rho\left(V_{i}-W_{i}\right)\right)=0
$$

(b) Momentum: Using an approach similar to that for continuity, the Reynolds transport theorem leads to the global momentum equation for the control volume $\Omega(t)$;

$$
\int_{\Omega(t)} \rho G_{i} \mathrm{~d} \Omega+\int_{\Omega(t)} \frac{\partial \sigma_{j i}}{\partial x_{j}} \mathrm{~d} \Omega=\int_{\Omega(t)} \rho \frac{\partial V_{i}}{\partial t} \mathrm{~d} \Omega+\int_{\Omega(t)} \rho\left(V_{j}-W_{j}\right) \frac{\partial V_{i}}{\partial x_{j}} \mathrm{~d} \Omega
$$

Thus, eqn (8) yields the pointwise momentum equation with respect to an arbitrarily moving grid point as:

$$
\rho G_{i}+\frac{\partial \sigma_{j i}}{\partial x_{j}}=\left.\rho \frac{\partial V_{i}}{\partial t}\right|_{\chi}+\rho\left(V_{i}-W_{i}\right) \frac{\partial V_{i}}{\partial x_{j}}
$$


Here $\sigma_{i j}$ are the Cauchy stress tensor components and $G_{i}$ are the components of body force per unit mass.

(c) Energy: Reynolds transport theorem for energy balance results in a global form of the energy equation as:

$$
\int_{\Omega(t)}\left[\rho \frac{\partial \hat{u}}{\partial t}+\rho\left(V_{i}-W_{i}\right) \frac{\partial \hat{u}}{\partial x_{j}}-\sigma_{i j} \frac{\partial V_{i}}{\partial x_{j}}+\frac{\partial q_{i}}{\partial x_{i}}-f\right] \mathrm{d} \Omega=0
$$

which subsequently reduces to the pointwise energy balance equation.

$$
\left.\rho \frac{\partial \hat{u}}{\partial t}\right|_{\boldsymbol{\chi}}+\rho\left(V_{i}-W_{i}\right) \frac{\partial \hat{u}}{\partial x_{j}}=\sigma_{j i} \frac{\partial V_{i}}{\partial x_{j}}-\frac{\partial q_{i}}{\partial x_{i}}+f
$$

In eqn (11) $\hat{u}$ is the specific internal energy, $\mathbf{q}$ is the heat flow vector and $f$ refers to the rate of heat addition by a source.

The internal energy $\hat{u}$ may simply be written as:

$$
\hat{u}=C_{p}\left(\theta-\theta_{\text {ref }}\right)
$$

where $\theta$ is the absolute temperature and $\theta_{\text {ref }}$ is some reference temperature.

Noting that the divergence of the heat flow vector may be related to the temperature gradient through Fourier's law of heat conduction and also that the symmetry of the Cauchy stress yields the relation

$$
\sigma_{j i} \frac{\partial V_{i}}{\partial x_{j}}=\sigma_{i i} D_{j i}
$$

where $\mathbf{D}$ is the rate of deformation tensor, the resulting pointwise energy balance equation has the form:

$$
\left.\rho C_{p} \frac{\partial \theta}{\partial t}\right|_{\chi}+\rho C_{p}\left(V_{i}-W_{i}\right) \frac{\partial \theta}{\partial x_{j}}=\sigma_{j i} D_{j i}+\frac{\partial}{\partial x_{i}}\left(k_{i j} \frac{\partial \theta}{\partial x_{j}}\right)+f
$$

where $k_{i j}$ is the heat conductivity of solid.

The time derivatives of density, stress and temperature in eqns (7), (9) and (14) represent the rates of the respective variables at a fixed grid point, identified by $\chi$. Depending on the value of $\mathbf{W}$ in these equations, different descriptions may be obtained.

(1) When $\mathbf{W}=\mathbf{V}$, the grid point moves with the material point and hence the description is Lagrangian.

(2) When $\mathbf{W}=0$, the grid point is fixed in space and therefore this corresponds to the Eulerian description.

Comparing the continuity, momentum and energy equations in the ALE description with the respective Lagrangian forms obtained by the substituting $\mathbf{W}=\mathbf{V}$ in eqns (7), (11) and (18), the following relations between time derivatives may be inferred:

$$
\begin{aligned}
& \left.\frac{\partial \rho}{\partial t}\right|_{\mathbf{x}}=\left.\frac{\partial \rho}{\partial t}\right|_{x}+\left(V_{i}-W_{i}\right) \frac{\partial \rho}{\partial x_{i}} \\
& \left.\frac{\partial V_{i}}{\partial t}\right|_{\mathbf{x}}=\left.\frac{\partial V_{i}}{\partial t}\right|_{x}+\left(V_{j}-W_{j}\right) \frac{\partial V_{i}}{\partial x_{j}} \\
& \left.\frac{\partial \theta}{\partial t}\right|_{\mathbf{x}}=\left.\frac{\partial \theta}{\partial t}\right|_{x}+\left(V_{i}-W_{i}\right) \frac{\partial \theta}{\partial x_{i}}
\end{aligned}
$$

A similar relation may also be establised for stress rates see [4] as:

$$
\left.\frac{\partial \sigma_{j i}}{\partial t}\right|_{\mathbf{x}}=\left.\frac{\partial \sigma_{j i}}{\partial t}\right|_{\boldsymbol{x}}+\left(V_{k}-W_{k}\right) \frac{\partial \sigma_{j i}}{\partial x_{k}}
$$

The above eqns (15)-(18) represent the relations between the material derivatives at a material point $\mathbf{X}$ and the derivatives at a grid point $\chi$. For problems of solid mechanics, where 
material properties are history dependent, the above set of equations provide the necessary link between the time derivatives, for the solution variables to be computed at the grid points.

\subsection{Principle of virtual work}

The equation of principle of virtual work may be derived by multiplying eqn (9) by an admissible virtual, material point displacement $\bar{u}$ and integrating over the current grid volume $\Omega(t)$. Different physical situations require different types of manipulations of the boundary points. For example, in most cases boundary displacements and tractions are prescribed on material points. In such cases it is appropriate to choose the corresponding grid points as Lagrangian. The entire boundary is an union of displacement boundary $\left(\Gamma_{U}\right)$ and the remaining boundary, including contact boundary, $\left(\Gamma_{T}\right)$ i.e. $\Gamma(t)$ is $\Gamma_{i U}(t) \cup \Gamma_{i T}(t)$ for $1 \leqslant i \leqslant 3$, where

$$
u_{i}=g_{i} \text { on } \Gamma_{i U}(t)
$$

and

$$
\sigma_{i i} n_{j}=T_{i} \quad \text { on } \Gamma_{i T}(t)
$$

With all the above assumptions, the principle of virtual work becomes

$$
\int_{\Omega(t)} \sigma_{j i} \frac{\partial \bar{u}_{i}}{\partial x_{j}} \mathrm{~d} \Omega+\int_{\Omega(t)} \rho \frac{\partial V_{i}}{\partial t} \bar{u}_{i} \mathrm{~d} \Omega+\int_{\Omega(t)} \rho\left(V_{j}-W_{j}\right) \frac{\partial V_{i}}{\partial x_{j}} \bar{u}_{i} \mathrm{~d} \Omega=\int_{\Omega(t)} \rho G_{i} \bar{u}_{i} \mathrm{~d} \Omega+\int_{\Gamma(t)} T_{i} \bar{u}_{i} \mathrm{~d} \Gamma
$$

The primary kinematic variable in (21) is displacement, and this equation is used for the solution of the incremental displacement discussed next.

Remark. For modelling an entire solid body with its boundaries, the boundary of the grid has to be properly represented. Such circumstances call for an assumption that the material boundary never leaves the grid boundary i.e.

$$
(\mathbf{V}-\mathbf{W}) \cdot \mathbf{n}=\mathbf{0} \text { on } \Gamma(t)
$$

where $\mathbf{n}$ is an outward normal to the boundary $\Gamma(t)$.

In this analysis it has been assumed that the motion of the material with respect to the grid points is quasi-static so that the transient term $\partial \mathrm{V} /\left.\partial t\right|_{\chi}=0$ in this analysis. The above assumption is logical within the realm of slow sheet metal forming processes with no impact.

\subsection{An iterative incremental algorithm for solving the equilibrium equation}

Nonlinearities in the equation of principle of virtual work (21) arise from (a) the convective term, (b) kinematic relations and (c) the material constitutive relations. This calls for an implicit iterative scheme for solution, to avoid accumulation of gross and undetectable errors and solution instabilities that might arise from the explicit methods, as pointed out in $[6,7]$. The commonly used family of one step algorithms using one parameter has been utilized in this analysis for first order system of differential equations. The stability behavior of the commonly used algorithms namely the generalized trapezoidal family or the generalized mid-point family have shown that both the one parameter $(\alpha)$ algorithms yield unconditional stability for $\alpha \geqslant \frac{1}{2}$ for linear problems $[6,24]$. However, as shown by Hughes $[24,25]$, the trapezoidal family gives unconditional stability only for $\alpha=1$ for certain nonlinear problems whereas it is second order accurate only for $\alpha=\frac{1}{2}$. On the other hand, the mid-point algorithm is unconditionally stable for $\alpha \geqslant \frac{1}{2}$ and second order accurate for $\alpha=\frac{1}{2}$ for the nonlinear problems. The integration scheme using the one parameter integration rule requires evaluation of gradients at time $t_{n+\alpha}$ in the Lagrangian-Eulerian formulation. In order to evaluate these gradients using the finite element method, various variables in the intermediate configuration are desired. Another criterion vital to the selection of the algorithm, which was pointed out by Hughes [25], is that an incrementally objective algorithm for integrating the stress rates causes no straining for rigid body rotations. This is achieved with maximum accuracy by evaluating the displacement gradients at the mid-step. All of the above considerations lead to the choice of the generalized mid-point strategy for analysis, particularly with $\alpha=\frac{1}{2}$.

The equation of principle of virtual work at an intermediate configuration $\Omega\left(t_{n+\alpha}\right)$ for 
$0 \leqslant \alpha \leqslant 1$ may thus be written as:

$$
\int_{\Omega\left(t_{n+\alpha}\right)} \sigma_{j i} \frac{\partial \bar{u}_{i}}{\partial x_{j}} \mathrm{~d} \Omega+\int_{\Omega\left(t_{n+\alpha}\right)} \rho\left(V_{j}-W_{j}\right) \frac{\partial V_{i}}{\partial x_{j}} \bar{u}_{i} \mathrm{~d} \Omega=\int_{\Omega\left(t_{n+\alpha)}\right.} \rho G_{i} \bar{u}_{i} \mathrm{~d} \Omega+\int_{\Gamma\left(t_{n+\alpha}\right)} T_{i} \bar{u}_{i} \mathrm{~d} \Gamma
$$

where all the variables are at an intermediate grid configuration with its position given by

$$
\mathbf{x}^{n+\alpha}=(1-\alpha) \mathbf{x}^{n}+\alpha \mathbf{x}^{n+1}
$$

Remark 1. Equation (23) may be solved together with the boundary constraint condition

$$
\left(\mathbf{V}^{n+\alpha}-\mathbf{W}^{n+\alpha}\right) \cdot \mathbf{n}^{n+\alpha}=0
$$

whenever the entire solid body has to be modeled. This condition may be imposed locally at boundary nodes.

Remark 2. The variables in (23), for example, stress, material velocity, grid velocity, density, body force etc. at the mid-step are related to their values at times $t_{n}$ and $t_{n+1}$ by the relation

$$
\beta^{n+\alpha}=(1-\alpha) \beta^{n}+\alpha \beta^{n+1}=\beta^{n}+\alpha \Delta^{g} \beta
$$

where $\beta$ may be any order tensor variable in eqn (23). A major observation that is to be made here, is that the increment $\Delta^{g} \beta$ is at a fixed grid point. This increment $\Delta^{g}$ of material path dependent variables like Cauchy stress, material velocity, density etc, thus has to be related to the increments $\Delta^{m}$ at a fixed material point. To obtain this relation, we start with an expression for time derivatives similar to eqns (15)-(18).

$$
\left.\frac{\partial \beta}{\partial t}\right|_{\chi}=\left.\frac{\partial \beta}{\partial t}\right|_{\mathbf{x}}+\left(W_{k}-V_{k}\right) \frac{\partial \beta}{\partial x_{k}}
$$

Equation (27) may be integrated over a time step $\Delta t$ between $t_{n}$ and $t_{n+1}$ using the one parameter integration rule to yield

$$
\Delta^{g} \beta=\Delta^{m} \beta+\Delta t\left(W_{k}^{n+\alpha}-V_{k}^{n+\alpha}\right) \frac{\partial \beta^{n+\alpha}}{\partial x_{k}^{n+\alpha}}
$$

The significance of this equation may be understood with reference to Fig. 2. A material point that coincides with a grid point in the intermediate configuration $\Omega\left(t_{n+\alpha}\right)$ does not necessarily coincide with the same grid point in the configurations $\Omega\left(t_{n}\right)$ and $\Omega\left(t_{n+1}\right)$. The

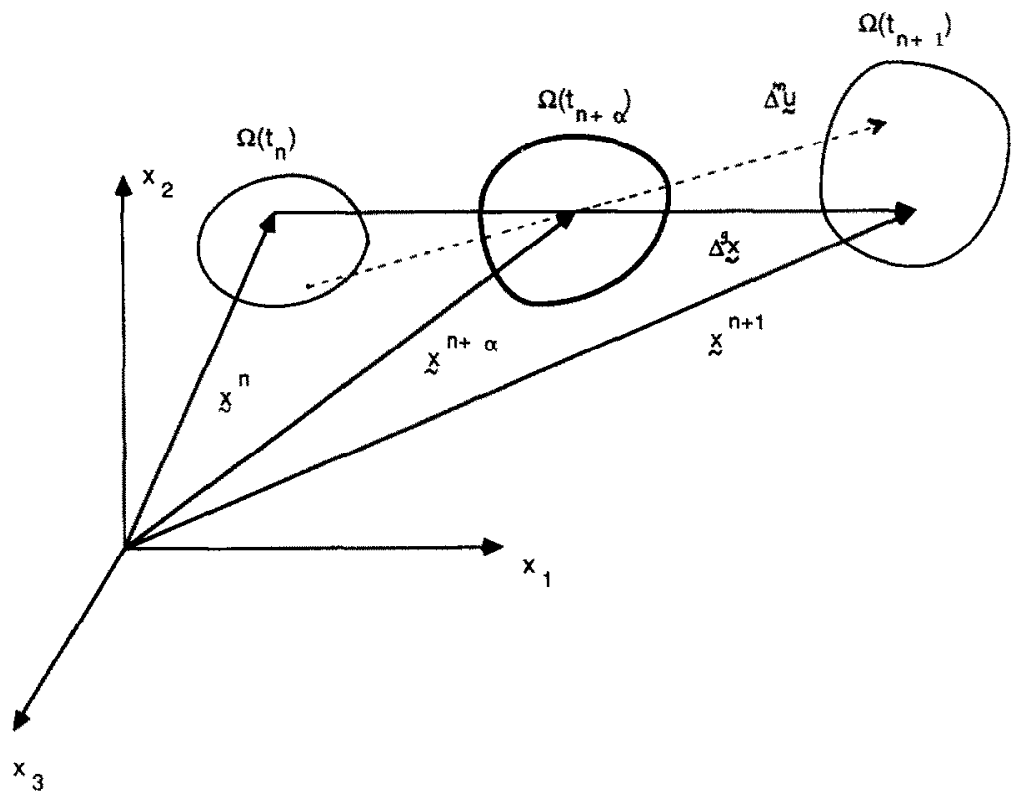

Fig. 2. The motion of grid points and material points in a time step. 
incremental values $\Delta^{m} \beta$ implies in equation correspond to those, for a material point which coincides with the grid point of interest at the intermediate configuration.

Remark 3. Using (28), the increments $\Delta^{g}$ of the material variables, $\sigma, \mathbf{V}, \rho$ etc. may be evaluated. From Fig. 2 and with the integration rule,

$$
\begin{aligned}
& \mathbf{W}^{n+\alpha}=\frac{\Delta^{g} \mathbf{x}}{\Delta t} \\
& \mathbf{V}^{n+\alpha}=\frac{\Delta^{m} \mathbf{u}}{\Delta t}
\end{aligned}
$$

Thus, the values of Cauchy stress, material velocity and density may be updated to the configuration at time $t_{n+1}$ by the relations:

$$
\begin{aligned}
\sigma_{i j}^{n+1} & =\sigma_{i j}^{n}+\Delta^{m} \sigma_{i j}+\left(\Delta^{g} x_{k}-\Delta^{m} u_{k}\right) \frac{\partial \sigma_{i j}^{n+\alpha}}{\partial x_{k}^{n+\alpha}} \\
V_{i}^{n+1} & =V_{i}^{n}+\frac{1}{\alpha}\left[\frac{\Delta^{m} u_{i}}{\Delta t}-V_{i}^{n^{*}}\right]+\left(\Delta^{g} x_{k}-\Delta^{m} u_{k}\right) \frac{\partial V_{i}^{n+\alpha}}{\partial x_{k}^{n+\alpha}} \\
\rho^{n+1} & =\rho^{n}+\Delta^{m} \rho+\left(\Delta^{g} x_{k}-\Delta^{m} u_{k}\right) \frac{\partial \rho^{n+\alpha}}{\partial x_{k}^{n+\alpha}}
\end{aligned}
$$

where $V_{i}^{n^{*}}$ is the velocity of a material point at time $t_{n}$ which coincides with the representative grid point in the intermediate configuration.

Remark 4. Even though eqn (30) may be used for the density increment, a more direct way of solving for the density at the grid points is through the use of the weak form of the continuity equation. Multiplying both sides of eqn (7) by a virtual density $\bar{\rho}$ and integrating over $\Omega\left(t_{n+\alpha}\right)$ the resulting weak form takes the form:

$$
\left.\int_{\Omega\left(t_{n+\alpha}\right)} \frac{\partial \rho}{\partial t}\right|_{\chi} \bar{\rho} \mathrm{d} \Omega+\int_{\Omega\left(t_{n+\alpha}\right)} \frac{\partial}{\partial x_{i}}\left\{\rho\left(V_{i}-W_{i}\right)\right\} \bar{\rho} \mathrm{d} \Omega+\int_{\Omega\left(t_{n+\alpha}\right)} \rho \frac{\partial W_{i}}{\partial x_{i}} \bar{\rho} \mathrm{d} \Omega=0
$$

When an entire solid body is to be modeled with its boundaries, the constraint condition (25) may be applied to eqn (31) to yield:

$$
\left.\int_{\Omega\left(t_{n+\alpha}\right)} \frac{\partial \rho}{\partial t}\right|_{\chi} \bar{\rho} \mathrm{d} \Omega=\int_{\Omega\left(t_{n+\alpha}\right)} \rho\left(V_{i}-W_{i}\right) \frac{\partial \bar{\rho}}{\partial x_{i}} \mathrm{~d} \Omega-\int_{\Omega\left(t_{n+\alpha}\right)} \rho \frac{\partial W_{i}}{\partial x_{i}} \bar{\rho} \mathrm{d} \Omega
$$

The above equation may be solved for $\left(\partial \rho /\left.\partial t\right|_{x}\right)^{n+\alpha}$ from which $\rho^{n+\alpha}$ is evaluated as:

$$
\rho^{n+\alpha}=\rho^{n}+\alpha \Delta t\left(\left.\frac{\partial \rho}{\partial t}\right|_{x}\right)^{n+\alpha}
$$

An iterative solution of eqns (32) and (33) is required, to solve for the density at the intermediate configuration.

\subsection{Implicit iterative scheme for solving the weak form of the energy balance equation}

Evaluation of the stress increments in the coupled thermo-mechanical process requires evaluation of temperature increments. The weak form of the energy balance equation is solved for the temperature increments using the mid-point strategy, for reasons that have been discussed in the preceeding section. The weak form is obtained by multiplying both sides of eqn (14) by an arbitrary virtual temperature $\bar{\theta}$ and integrating over the mid-step grid volume which yields:

$$
\begin{aligned}
\left.\int_{\Omega\left(t_{n+\alpha}\right)} \rho C_{p} \frac{\partial \theta}{\partial t}\right|_{\chi} \bar{\theta} \mathrm{d} \Omega+\int_{\Omega\left(t_{n+\alpha}\right)} \rho C_{p}\left(V_{i}-W_{i}\right) \frac{\partial \theta}{\partial x_{i}} \bar{\theta} \mathrm{d} \Omega+\int_{\Omega\left(t_{n+\alpha}\right)} k_{i j} \frac{\partial \theta}{\partial x_{j}} \frac{\partial \bar{\theta}}{\partial x_{i}} \mathrm{~d} \Omega \\
=\int_{\Omega\left(t_{n+\alpha}\right)} \sigma_{j i} D_{j i} \bar{\theta} \mathrm{d} \Omega+\int_{\Omega\left(t_{n+\alpha}\right)} q_{n} \bar{\theta} \mathrm{d} \Omega+\int_{\Gamma^{T}\left(t_{n+\alpha}\right)} f \bar{\theta} \mathrm{d} \Gamma
\end{aligned}
$$

The boundary of the grid configuration may be assumed to be the sum of two basic types of 
boundaries i.e. $\Gamma^{T}(t)$ is $\Gamma_{1}^{T}(t) \cup \Gamma_{2}^{T}(t)$, where

and

$$
\theta=\theta_{g} \quad \text { on } \Gamma_{1}^{T}(t)
$$

$$
k_{i j} \frac{\partial \theta}{\partial x_{j}} n_{i}=q_{n} \text { on } \Gamma_{2}^{T}(t)
$$

Remark 1. In the event where the temperature is prescribed on material points, the corresponding nodes may be made Lagrangian.

Remark 2. The heat flow vector may be assumed to be a combination of flux due to convection between environment and the body, radiation from the environment, heat transferred between the workpiece and the die, and heat generated by friction.

Remark 3. The rate of deformation tensor is generally decomposed into a thermoelastic part and viscoplastic part. Of the viscoplastic deformation, only a fraction is responsible for heat generation, the remainder being used for the change in internal energy due to dislocation density change, change of grain boundaries and phase changes etc.

Coming back to eqn (34), the rate of temperature change at a grid point may be approximated as:

$$
\left(\left.\frac{\partial \theta}{\partial t}\right|_{\chi}\right)^{n+\alpha}=\frac{\Delta^{g} \theta}{\Delta t}
$$

Noting that the variables at midstep are unknown, eqn (34) has to be solved iteratively using the equation

$$
\begin{aligned}
\frac{1}{\Delta t} \int_{\Omega\left(t_{n+\alpha}\right)} \rho C_{p} \Delta^{8} \theta \bar{\theta} \mathrm{d} \Omega= & \int_{\Omega\left(t_{n+\alpha}\right)} \sigma_{i i} D_{j i} \bar{\theta} \mathrm{d} \Omega+\int_{\Omega\left(t_{n+\alpha}\right)} f \bar{\theta} \mathrm{d} \Omega \\
& +\int_{\Gamma_{2}^{T}\left(t_{n+\alpha}\right)} q_{n} \bar{\theta} \mathrm{d} \Gamma-\int_{\Omega\left(t_{n+\alpha}\right)} \rho C_{p}\left(V_{i}-W_{i}\right) \frac{\partial \theta}{\partial x_{i}} \bar{\theta} \mathrm{d} \Omega \\
& -\int_{\Omega\left(t_{n+\alpha}\right)} k_{i j} \frac{\partial \theta}{\partial x_{j}} \frac{\partial \bar{\theta}}{\partial x_{i}} \mathrm{~d} \Omega
\end{aligned}
$$

Equation (37) has to be solved simultaneously with eqn (23) because of the coupling of the terms involving stress and temperature and an iterative algorithm is described later.

\section{CONSTITUTIVE RELATIONS}

Hot sheet metal forming of metals is carried out at high temperatures. At elevated temperatures, most material behavior is governed by plastic as well as rheologic effects. In this analysis, the material is modeled as thermoelastic-viscoplastic, where viscous effects become pronounced after the material has yielded plastically. Initial anisotropy is incorporated to account for the grain orientation effects. The material is also assumed to be strain-hardening and temperature dependent.

\subsection{Stress Rate}

The principle of material frame indifference is embodied in the constitutive theory by the requirement that only objective tensor fields must enter the constitutive relations. In large deformation analysis, the use of the objective Jaumann's rate has been fairly standard, till it was found by Nagtegaal and de Jong [10] to give rise to oscillatory stress response in simple shear and kinematic hardening. Johnson and Bammann [11] recommended a remedy by using a rate which is more physical in the sense, that the time derivative is taken, after extracting rigid body rotation from the deformation. Such a rate was introduced by Green and Naghdi [12]. The rotated Cauchy stress is defined as:

$$
\mathbf{t}=\mathbf{R}^{T} \boldsymbol{\sigma R}
$$

where $\boldsymbol{\sigma}$ is the Cauchy stress tensor, and $\mathbf{R}$ is a proper orthogonal tensor representing pure rotation obtained from the $\mathbf{R U}$ decomposition of the deformation gradient $\mathbf{F}$. 
Remark. The rate of rotated Cauchy stress tensor remains invariant under rigid body motions i.e. $\dot{t}^{+}=\dot{t}$. In the case of anisotropic materials, the above property is desirable, because it gives rise to form invariant constitutive relations. A second advantage of using this stress is that the yield function for anisotropic materials remain unchanged under rigid body motion. On account of these advantages the use of the rotated Cauchy stress in the constitutive relation for anisotropic pre-rolled sheet metals seem inevitable.

\subsection{Constitutive Relations for a Thermoelastic-Viscoplastic Materials with Initial Anisotropy}

The commonly employed relation between an objective stress rate and the rate of deformation tensor for an elastic process is of the form:

$$
\check{\mathbf{\sigma}}=\mathbf{f}(\boldsymbol{\sigma}, \mathbf{D})
$$

The frame indifference requirement restricts the function $\mathbf{f}$ to be isotropic in $\mathbf{D}$ which makes it unsuitable for anisotropic materials. Another argument regarding the use of a Cauchy stress rate-rate of deformation type relation was pointed out by Nagtegaal [13]. He showed that the integration of the rate of deformation tensor depends on the rigid body rotation of the material and thus, is not a pure strain measure. On the other hand, the rotated rate of deformation tensor when integrated yields a purely strain measure. These considerations have prompted the choice of a constitutive relation of the form:

$$
\dot{\mathbf{t}}=\mathbf{F}(\mathbf{t}, \mathbf{d})
$$

where $\mathbf{d}=\mathbf{R}^{T} \mathbf{D R}$ is the rotated rate of deformation tensor. This relation was introduced by Green and McInnis [28]. However the above eqn (40) is not suitable for hyperelastic materials as shown in $[29,11]$.

The rate of deformation tensor may be assumed to undergo an additive decomposition into an elastic, thermal and viscoplastic part, for which a thermodynamic argument may be found within the framework of internal variable theory as discussed by Pinsky et al. [9]. Then the rotated rate of deformation also admits the decomposition of the form

where

$$
\mathbf{d}=\mathbf{d}^{e}+\mathbf{d}^{\text {th }}+\mathbf{d}^{v p}
$$

$$
\begin{aligned}
\mathbf{d}^{e} & =\mathbf{R}^{T} \mathbf{D}^{e} \mathbf{R} \\
\mathbf{d}^{t h} & =\mathbf{R}^{T} \mathbf{D}^{t h} \mathbf{R} \\
\mathbf{d}^{v p} & =\mathbf{R}^{T} \mathbf{D}^{v p} \mathbf{R}
\end{aligned}
$$

In order to model the viscoplastic rate of deformation tensor, it is necessary to find a proper static yield criterion for initially anisotropic, work hardening materials.

\subsubsection{A static yield criterion for initially anisotropic, work hardening materials}

The directional properties inherent to sheet metals, require the inclusion of anisotropy in the static yield criterion of the material. In 1948, Hill generalized von Mises yield criterion to include anisotropy [14] which was later extended for strain hardening by $\mathrm{Hu}$ [15]. The yield criterion suggested by the above authors has been extended for large deformation problems.

It has been shown by Green and Naghdi [12] and Johnson and Bammann [11] that a yield function which is a function of Cauchy stress is not appropriate for anisotropic materials due to the fact that they are altered under superposed rigid body motions. As an alternative, a yield function of the form:

$$
f\left(\mathbf{t}, \mathbf{E}^{p}, \theta\right)=\kappa\left(W_{p}, \theta\right)
$$

is suggested, where $\mathbf{E}^{p}$ is a Lagrangian plastic strain measure, $\theta$ is the temperature and $\kappa\left(W_{p}, \theta\right)$ is a work-hardening parameter. Since the rotated Caucy stress is invariant under superposed rigid body motions, eqn (41) is invariant under such motion and is suitable for general anisotropic materials.

An effective rotated Cauchy stress is defined as:

$$
\bar{t}^{2}=\frac{1}{2}\left[F\left(t_{11}-t_{22}\right)^{2}+G\left(t_{22}-t_{33}\right)^{2}+H\left(t_{33}-t_{11}\right)^{2}+6 L t_{12}^{2}+6 M t_{23}^{2}+6 N t_{31}^{2}\right]
$$


where $F, G, H, L, M$ and $N$ are parameters of anisotropy dependent on the state of strain and the temperature. In eqn (43) directions 1,2 and 3 correspond to the principal axes of anisotropy in the unrotated configuration. In sheet metal forming problems we shall assume the principal material axes to remain orthogonal in the unrotated configuration, throughout the deformation process. It is also assumed that the material is transversely isotropic and rotational isotropy exists in the mid-plane of the sheet metal. Under these assumptions, the effective stress becomes

$$
\begin{aligned}
\bar{t}^{2}= & \frac{1}{2}\left[(F+G) t_{11}^{2}+(F+G) t_{22}^{2}+2 G t_{33}^{2}-2 F t_{11} t_{22}-2 G t_{22} t_{33}\right. \\
& \left.-2 G t_{11} t_{33}+2(G+2 F) t_{12}^{2}+6 M t_{23}^{2}+6 M t_{31}^{2}\right] \\
= & \{\mathbf{t}\}^{T}[\mathbf{A}]\{\mathbf{t}\}
\end{aligned}
$$

where $\{\mathbf{t}\}^{T}=\left\{t_{11} t_{22} t_{33} t_{12} t_{23} t_{31}\right\}$ and

$$
[A]=\frac{1}{2}\left[\begin{array}{cccccc}
F+G & -F & -G & 0 & 0 & 0 \\
-F & F+G & -G & 0 & 0 & 0 \\
-G & -G & 2 G & 0 & 0 & 0 \\
0 & 0 & 0 & 2(G+2 F) & 0 & 0 \\
0 & 0 & 0 & 0 & 6 M & 0 \\
0 & 0 & 0 & 0 & 0 & 6 M
\end{array}\right]
$$

Parameters for anisotropy. (a) Initial yield: The parameters of anisotropic yield criterion arc determined by tension tests in the 1 and 3 direction and a shear test in the 2-3 plane. By letting all stresses except the one under consideration to be equal to zero, the initial yield parameters $F_{0}, G_{0}$ and $M_{0}$ are obtained as:

$$
\begin{aligned}
F_{0} & =\bar{t}_{0}^{2}\left[\frac{2}{t_{10}^{2}}-\frac{1}{t_{30}^{2}}\right] \\
G_{0} & =\frac{\bar{t}_{0}^{2}}{t_{30}^{2}} \\
M_{0} & =\frac{1}{3 t_{230}^{2}}
\end{aligned}
$$

where $t_{10}, t_{30}$ and $t_{230}$ are the initial yield stresses in the tension and shear in the respective direction. If the reference direction coincides with the 1 direction, $\bar{t}_{0}$ may be chosen to be $t_{10}$ and the parameters will adjust accordingly.

(b) Subsequent yield (strain-hardening): The uniaxial static yield stresses for a strain hardening material changes with increasing plastic deformation. The parameters in the effective stress expression for anisotropic materials are assumed to depend on the plastic work done in a static process in which rate effects are absent. These parameters represent the work hardening effect in a rate independent elastic-plastic process. Their evaluation is based on an assumption that for an equivalent change in the effective stress, the plastic work done each direction should be the same. The plastic work may be expressed as:

$$
W_{p}=\int_{t_{1}}^{t_{2}} \bar{\sigma}_{s} \bar{D}^{p} \mathrm{~d} t=\int_{t_{1}}^{t_{2}} \bar{t}_{s} \bar{d}^{p} \mathrm{~d} t=\int_{t_{1}}^{t_{2}}\left(t_{i j}\right)_{s} d_{i j}^{p} \mathrm{~d} t
$$

where a subscript $s$ corresponds to a static condition. The total equivalent plastic rotated strain may be written as:

$$
\hat{\boldsymbol{\epsilon}}^{p}=\int_{0}^{t} d^{p} \mathrm{~d} t
$$



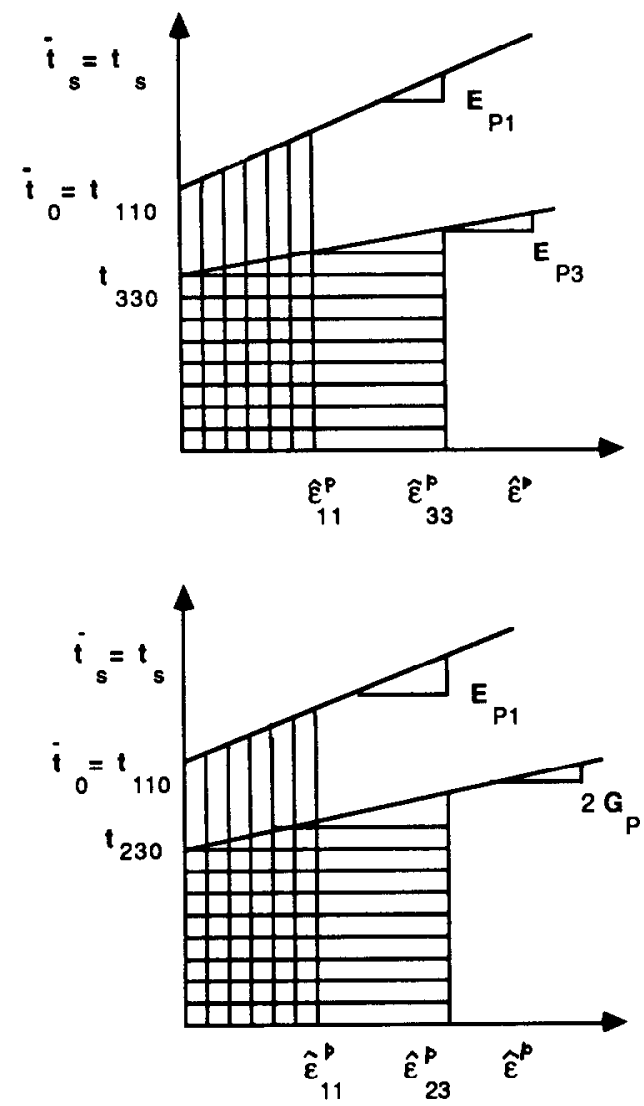

Fig. 3. Equating plastic work.

Then the parameters are obtained from Fig. 3 as:

$$
\begin{array}{r}
F+G=\frac{2 \bar{t}_{s}^{2}}{\frac{E_{p 1}}{E_{p}}\left(\bar{t}_{s}^{2}-\bar{t}_{0}^{2}\right)+t_{10}^{2}} \\
G=\frac{\bar{t}_{s}^{2}}{\frac{E_{p 3}}{E_{p}}\left(\bar{t}_{s}^{2}-\bar{t}_{0}^{2}\right)+t_{30}^{2}} \\
3 M=\frac{\bar{t}_{s}^{2}}{\frac{G_{p}}{E_{p}}\left(\bar{t}_{s}^{2}-\bar{t}_{0}^{2}\right)+t_{230}^{2}}
\end{array}
$$

where $\bar{t}_{s}, \bar{t}_{0}, t_{10}, t_{30}$ and $t_{230}$ are the equivalent current maximum stress, the initial equivalent yield stress, the uniaxial initial yield stresses in 1 and 3 directions and the initial yield strength in shear in the 2-3 plane respectively. The yield strengths correspond to the static yield condition. Also $E_{p}, E_{p 1}, E_{p 3}$ and $G_{p}$ are the slopes of the equivalent stress-plastic strain curve, stress-plastic strain in the 1 and 3 directions and the shear stress-plastic shear strain curve in the 2-3 plane. The equivalent yield strength may be made to be the same as the yield strengths in any of the directions and the parameters may be adjusted accordingly. The initial yield strengths are all temperature dependent.

\subsubsection{Thermal rate of deformation}

The thermal rate of deformation follows directly from the relations used for small deformation problems as:

$$
D_{i j}^{i h}=\alpha_{i j} \dot{\theta}
$$


where $\dot{\theta}$ is the rate of temperature change and $\alpha_{i j}$ is a temperature dependent coefficient of thermal expansion. Assuming that $\alpha_{i j}$ is a constant isotropic tensor in this analysis, the rotated rate of thermal deformation becomes

$$
d_{i j}^{t h}=\alpha \delta_{i j} \dot{\theta}
$$

where $\delta_{i j}$ is the Kronecker delta. The rate of temperature change is obtained by solving the energy balance eqn (37).

\subsubsection{Visco-plastic rate of deformation}

The rate of viscoplastic strain introduced in this section follows from the concept of closest point mapping. It is assumed that the onset of viscoplastic behavior is governed by a static yield condition and the components of the viscoplastic strain rate tensor are functions of stresses in excess of the static yield strength. Such a model introduced by Perzyna [8] has been extended to account for large deformation. The model suffers the disadvantages of not being able to include rate dependent initial yield and time dependent strain recovery. Bauschinger effect is also not included in the model. However, this model has been chosen for the sake of simplicity. A yield function $F$ is used to characterize the material which may be expressed as:

$$
F\left(\mathbf{t}, \mathbf{d}^{v p}, \theta\right)=\bar{t}-\kappa\left(W_{p}, \theta\right)
$$

where $K$ is the work hardening parameter with values $\bar{t}_{0}$ for initial yield and $\bar{t}_{s}$ subsequently. The rotated rate of viscoplastic deformation tensor may then be written in the form

$$
d_{i j}^{v p}=\gamma<\Phi\left(\bar{t}-\kappa\left(W_{p}, \theta\right)\right)>\frac{\partial \bar{t}}{\partial t_{i j}}
$$

where $\gamma$ is a temperature dependent viscosity coefficient and \langle\rangle is the MacCauley operator. This model for viscoplastic rate of deformation satisfies the incompressibility condition i.e. $d_{k k}^{v p}=0$. Normality of this strain rate tensor to the dynamical yield surface $(\bar{t})$ in the rotated stress space is evident from its definition. The function $\Phi(F)$ may be chosen to take different forms depending on the material and conditions of the numerical experiment. A form $\Phi(F)=F^{p}$ has been chosen in the analysis for its proven suitability for metals.

In order to have a complete set of rate constitutive relations, it is important to supplement the stress-strain equation with evolution relations for internal variables. They may be of the form

$$
\dot{\mathbf{q}}=\mathbf{f}(\boldsymbol{\sigma}, \mathbf{q})
$$

where $\mathbf{q}$ are the spatial internal variables and $\mathbf{f}$ is a tensor function of stresses and internal variables. In this analysis the work hardening parameter $k$ has been chosen to be the internal variable and its rate may be defined as:

$$
\frac{\partial \kappa}{\partial t}=E_{p} \bar{d}^{v p}
$$

where $\bar{d}^{v p}$ is the effective viscoplastic rate of deformation. It is thus noted that the viscoplastic rate of deformation governs the change of parameter $\kappa$.

The effective rate of deformation tensor $\tilde{d}^{v p}$ is defined through the relation:

$$
\bar{t} \vec{d}^{v p}=t_{i j} d_{i j}^{v p}
$$

The incompressibility condition of the viscoplastic rate of deformation $\left(d_{k k}^{v p}=0\right)$, may be used to rewrite eqn (56) as:

$$
\bar{t} \bar{d}^{v p}=d_{11}^{v p}\left(t_{11}-t_{33}\right)+d_{22}^{v p}\left(t_{22}-t_{33}\right)+2 d_{12}^{v p} t_{12}+2 d_{23}^{v p t_{23}}+2 d_{31}^{v p} t_{31}
$$

Using the definition of the viscoplastic rotated rate of deformation (53) to eliminate the stresses $t_{i j}$ from eqn (57), an expression may be derived for the equivalent rotated rate of 
viscoplastic deformation $\bar{d}^{v p}$ as:

$$
\bar{d}^{v p}=\frac{2}{\gamma\left[\Phi\left(\bar{t}-K\left(W_{p}, \theta\right)\right)\right.}\left[\frac{1}{G+2 F}\left\{\left(d_{11}^{v p}\right)^{2}+\left(d_{22}^{v p}\right)^{2}+\frac{F}{G}\left(d_{33}^{v p}\right)^{2}+2\left(d_{12}^{v p}\right)^{2}\right\}+\frac{2}{3 M}\left\{\left(d_{23}^{v p}\right)^{2}+\left(d_{31}^{v p}\right)^{2}\right\}\right]
$$

To summarize, the entire set of rate constitutive equations to be used in the analysis are as follows.

$$
\begin{aligned}
\dot{\mathbf{t}} & =\mathbf{E}:\left(\mathbf{d}-\mathbf{d}^{t h}-\mathbf{d}^{v p}\right) \\
\frac{\partial K}{\partial t} & =E_{p} \bar{d}^{v p}
\end{aligned}
$$

where $E_{i j k l}$ is the fourth order elasticity tensor for a transversely isotropic material, accounting for the grain orientations in the sheet metal.

\section{INTEGRATION OF RATE CONSTITUTIVE EQUATIONS OF ELASTO- VISCOPLASTICITY}

An integration algorithm for rate constitutive equations should satisfy three basic requirements as pointed by Pinsky et al. [9].

(1) Consistency with the constitutive equations.

(2) Numerical stability.

(3) Incremental objectivity.

The first two conditions when satisfied assure the convergence of the integration scheme and the third condition satisfies the physical requirement that the algorithm is invariant with respect to superposed rigid body motion. A number of algorithms which fall in the general category of return mapping algorithms satisfying the above conditions, have been proposed; namely the radial return algorithm [16, 17], the closest point mapping algorithm [9] etc. These methods have been found to be better in performance than the traditional initial load or tangent stiffness methods because of their ability to deal with corners in yield surfaces and also because of the relative ease of satisfying the consistency condition. A new class of the return mapping algorithms within the framework of the operator splitting methodology is considered in this analysis. This method proposed very recently by Ortiz et al. [30], is applicable to a very general class of plastic and viscoplastic constitutive models. The authors have proved that the algorithm provides a quadratic rate of convergence to the updated plastic state.

\subsection{The Operator Split and the Return Mapping Algorithm}

The return mapping algorithm is obtained as a consequence of the additive decomposition of the rotated rate of deformation tensor into the elastic, thermal and the viscoplastic parts as shown in eqn (41). The set of evolution equations are given as:

$$
\begin{aligned}
\mathbf{d} & =\mathbf{d}^{e}+\mathbf{d}^{\text {th }}+\mathbf{d}^{v p} \\
\dot{\mathbf{t}} & =\mathbf{E}: \mathbf{d}^{e} \\
\mathbf{d}^{t h} & =\alpha \dot{\theta} \mathbf{I} \\
\mathbf{d}^{v p} & =\gamma\left\langle\Phi\left(\bar{t}-\kappa\left(W_{p}, \theta\right)\right)\right\rangle \frac{\partial \bar{t}}{\partial \mathbf{t}} \\
\frac{\partial \kappa}{\partial t} & =E_{p} \bar{d}^{v p}
\end{aligned}
$$


The set of eqns (60) may be decomposed into elastic, thermal and viscoplastic parts as:

(a) Elastic:

$$
\begin{aligned}
\mathbf{d} & =\mathbf{d}^{e}+\mathbf{d}^{t h}+\mathbf{d}^{v p} \\
\dot{\mathbf{t}} & =\mathbf{E}: \mathbf{d} \\
\mathbf{d}^{\text {th }} & =0 \\
\mathbf{d}^{v p} & =0 \\
\frac{\partial K}{\partial t} & =0
\end{aligned}
$$

(b) Thermal:

$$
\begin{aligned}
\mathbf{d} & =\mathbf{d}^{e}+\mathbf{d}^{t h}+\mathbf{d}^{v p}=0 \\
\dot{\mathbf{t}} & =-\mathbf{E}: \mathbf{d}^{\text {th }} \\
\mathbf{d}^{t h} & =\alpha \dot{\theta} \mathbf{I} \\
\mathbf{d}^{v p} & =0 \\
\frac{\partial K}{\partial t} & =0
\end{aligned}
$$

(c) Viscoplastic:

$$
\begin{aligned}
\mathbf{d} & =\mathbf{d}^{e}+\mathbf{d}^{t h}+\mathbf{d}^{v p}=0 \\
\mathbf{i} & =-\mathbf{E}: \mathbf{d}^{v p} \\
\mathbf{d}^{t h} & =0 \\
\mathbf{d}^{v p} & =\gamma\left\langle\Phi\left(\bar{t}-\kappa\left(W_{p}, \theta\right)\right)\right\rangle \frac{\partial \bar{t}}{\partial \mathbf{t}} \\
\frac{\partial \kappa}{\partial t} & =E_{p} \bar{d}^{v p}
\end{aligned}
$$

The decoupled eqns (61), (62) and (63) may be interpreted in the following way. In the elastic eqn (61), the thermal and viscoplastic responses are frozen. The entire deformation is responsible for elastically straining the material. In the thermal equation, the stresses are relaxed due to the rate of temperature increase, in a rigid body since $d=0$. The viscoplastic response is still frozen. Finally the viscoplastic equation, represent a set of relaxation equations for stresses and work hardening parameter for a rigid body under isothermal conditions $(\dot{\theta}=0)$.

The additive structure of the material rate constitutive relations may be exploited in the finite element analysis for solving for stresses and displacements. The equation of principle of virtual work (23) after the implementation of the set of constitutive eqn (60) may be written as:

$$
\mathbf{A} \dot{\mathbf{x}}=\mathbf{B}(\mathbf{x})+\mathbf{f}
$$

where $\mathbf{A}$ is a coefficient matrix, $\mathbf{B}$ is a nonlincar operator and $\mathbf{x}$ is a one dimensional array consisting of displacements, stresses and internal variables. The decomposition of the constitutive equation gives rise to a splitting of the evolution operator $\mathbf{B}(\mathbf{x})$ into elastic, thermal and viscoplastic parts.

$$
\mathbf{B}=\mathbf{B}^{e}+\mathbf{B}^{t h}+\mathbf{B}^{v p}
$$

Thus, eqn (64) may be rewritten as

$$
\mathbf{A} \dot{\mathbf{x}}=\mathbf{B}^{e} \mathbf{x}+\mathbf{B}^{\text {th }} \mathbf{x}+\mathbf{B}^{v p}(\mathbf{x})
$$

A detailed construction of ths eqn (65) may be found in [31]. The product algorithm may now be applied as:

$$
\mathbf{F}(\mathbf{x})=\mathbf{F}^{v p}(\mathbf{x}) \mathbf{F}^{t h}(\mathbf{x}) \mathbf{F}^{e}(\mathbf{x})
$$


where $F^{e}(\mathbf{x})$ is an integration algorithm for solution of $\mathbf{A} \dot{\mathbf{x}}=\mathbf{B}^{e} \mathbf{x}, \mathbf{F}^{\text {th }}(\mathbf{x})$ is an algorithm for solving $\mathbf{A} \dot{\mathbf{x}}=\mathbf{B}^{\text {th }} \mathbf{x}$, and $\mathbf{F}^{v p}(\mathbf{x})$ is an algorithm for solving $\mathbf{A} \dot{\mathbf{x}}=\mathbf{B}^{v p}(\mathbf{x})$.

The product algorithm simply states that a solution algorithm for a thermoelastic-viscoplastic problem may be obtained by solving at each time step an elastic dynamic problem first, then applying a thermal algorithm to the solution vector of the elastic problem and finally applying a viscoplastic algorithm to the solution vector obtained from the thermal algorithm. The individual algorithms are briefly discussed next.

\subsubsection{Elastic algorithm}

In this algorithm, the principle of virtual work is solved in conjunction with the elastic constitutive relations (61). The requirements of numerical integration stated before, have been proven to be satisfied by the generalized mid point rule. Hughes [32] has proven this algorithm to be incrementally objective for $\alpha=\frac{1}{2}$. The rotated Cauchy stress may thus be integrated over a time step $\Delta t$, using the generalized mid-point rule to yield:

$$
\mathbf{t}^{n+1}-\mathbf{t}^{n}=\Delta \dot{t}^{n+\alpha} \quad 0 \leqslant \alpha \leqslant 1
$$

where $\dot{t}^{n+\alpha}=(\mathbf{E}: \mathbf{D})^{n+\alpha}$. Substituting this result to eqn (67), we obtain a relation between the increment of the rotated Cauchy stress and the rate of deformation, referred to an intermediate configuration as

$$
\Delta t_{i j}=\Delta t\left(E_{i j k l} R_{m k} R_{n l} D_{m n}\right)^{n+\alpha}
$$

In eqn (68) $\mathbf{R}$ is the rotation tensor and $\mathbf{D}$ is the rate of deformation tensor. To obtain second order accuracy and exclude the effects of rigid body rotation, the rate of deformation tensor is evaluated with respect to a mid-point configuration as shown in [18]. The rate of deformation tensor $\mathbf{D}^{n+\alpha}$, evaluated at an intermediate configuration may be approximated as:

$$
D_{i j}^{n+\alpha}=\frac{1}{2 \Delta t}\left[\frac{\partial \Delta^{m} u_{i}}{\partial x_{j}^{n+\alpha}}+\frac{\partial \Delta^{m} u_{j}}{\partial x_{i}^{n+\alpha}}\right]
$$

where $\Delta^{m}$ corresponds to the increment at a material point.

The increment of the Cauchy stress then follows from the relation $\left(\sigma_{n+1}=\mathbf{R}(\mathbf{t}+\Delta \mathbf{t}) \mathbf{R}^{T}\right)$ between the Cauchy stress and the rotated Cauchy stress to be

$$
\Delta^{m} \sigma_{j i}=R_{j k}^{n+1} t_{k l}^{n} R_{i l}^{n+1}+R_{j p}^{n+1} R_{i q}^{n+1} R_{r k}^{n+\alpha} R_{s l}^{n+\alpha} E_{p q k l} \frac{\partial \Delta^{m} u_{r}}{\partial x_{s}^{n+\alpha}}-\sigma_{j i}^{n}
$$

The above algorithm is consistent with rate constitutive relations and unconditionally stable for $\alpha \geqslant \frac{1}{2}$. Thermal and viscoplastic strains and the work hardening parameter $\kappa$ (radius of the static yield surface) remain unchanged in this phase.

\subsubsection{Thermal algorithm}

The stress obtained by the elastic algorithm are relaxed by integrating the rate eqns (62) directly, to give

$$
\left(t_{i j}^{n+1}\right)^{\imath h}-\left(t_{i j}^{n+1}\right)^{e}=-\alpha E_{i j k k} \Delta \theta
$$

It is to be noted that geometry of the deformed configuration, viscoplastic strain and work-hardening parameter remain unchanged in this algorithm.

\subsubsection{Viscoplastic algorithm}

The stress obtained from the solution of the thermal algorithm is now treated as the initial condition to the stress relaxation problem resulting from the set of viscoplastic eqns (63). The body is considered to be rigid and the material properties are temperature independent. For simplicity, in the power law model $\Phi(F)=F^{p}$, the exponent $p$ has been assumed to be 1 . It has been shown in [21], that at relatively low strain rates, the power strain rate law with an exponent 1 , closely simulates the behavior of metals when compared with experimental results. 
With this specification the rate dependent plastic relaxation equations become

$$
\begin{aligned}
& \frac{\partial t_{i j}}{\partial t}=-\gamma F E_{i j k l} \frac{\partial t}{\partial t_{k l}} \\
& \frac{\partial \kappa}{\partial t}=\gamma E_{p} F
\end{aligned}
$$

where $F=\bar{t}-\kappa\left(W_{p}\right)$ and viscoplastic loading $(F>0)$ has been implicitly assumed. The rate of change of the overstress $F$ during the relaxation process may be obtained by chain rule as:

$$
\frac{\partial F}{\partial t}=\frac{\partial F}{\partial t_{i j}} \frac{\partial t_{i j}}{\partial t}+\frac{\partial F}{\partial K} \frac{\partial K}{\partial t}
$$

After appropriate substitution from eqn (72) the rate of change of the yield surface becomes

$$
\frac{\partial F}{\partial t}=-\gamma F\left[E_{i j k l} \frac{\partial \bar{t}}{\partial t_{i j}} \frac{\partial \bar{t}}{\partial t_{k l}}+E_{p}\right]
$$

This equation represents the consistency condition for viscoplasticity. Defining an instantaneous relaxation time as:

$$
\tau=\frac{1}{\gamma\left[E_{i j k l} \frac{\partial \bar{t}}{\partial t_{i j}} \frac{\partial \bar{t}}{\partial t_{k l}}+E_{p}\right]}
$$

the rate of growth of the yield function takes the following form

$$
\frac{\partial F}{\partial t}=-\frac{F}{\tau}
$$

This equation determines the location of a stress state on the return path, which reaches the updated dynamic yield surface through a sequence of straight segments as shown in Fig. 4. In each of these straight segments (i) describing the return path for a time stcp $\Delta t$, the relaxation time is assumed to remain unchanged and have a value corresponding to the beginning of the segment. Then eqn (76) may be integrated in each segment to obtain

$$
\frac{F_{i+1}^{n+1}}{F_{i}^{n+1}}=e^{-\Delta t_{i} / \tau}
$$

where $\Delta t_{i}=t_{i+1}-t_{i}$ and $F_{i+1}^{n+1}$ corresponds to the yield surface at the end of the time segment. The integration over a time step is executed initially, by controlling the relaxation in which a

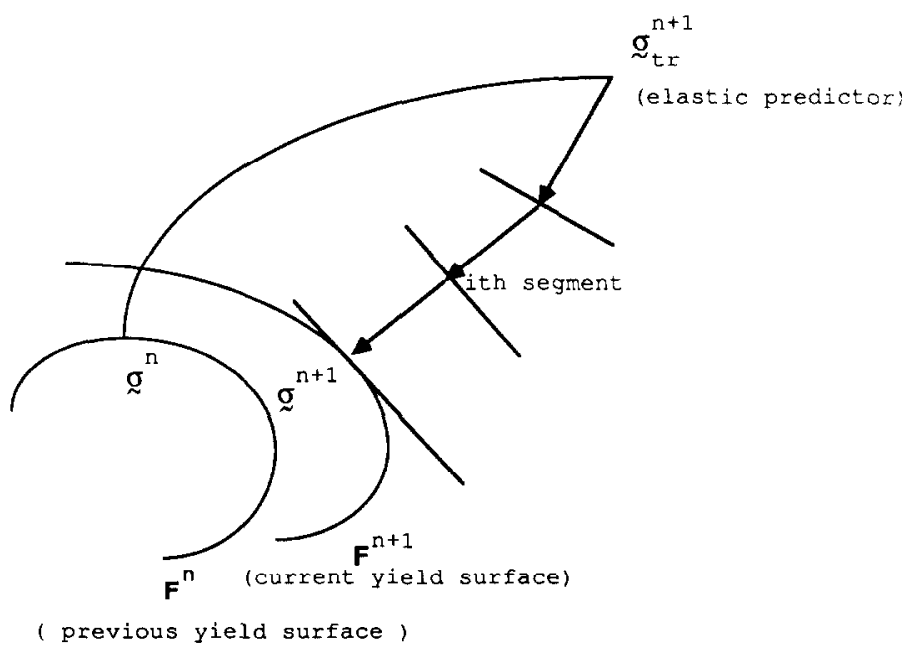

Fig. 4. Gcometric interpretation of the return mapping algorithm for viscoplasticity. The elastic predictor based on the elastic and thermal algorithm is brought to the current yield surface by relaxation over linear segments. 
parameter is defined as:

$$
\Delta \delta^{i}=\gamma F_{i}^{n+1} \tau_{i}
$$

The rate eqns (72) and (76) may then be integrated over a time segment to yield the following relaxation equations.

$$
\begin{aligned}
\left(t_{i j}\right)_{i+1}^{n+1} & =\left(t_{i j}\right)_{i}^{n+1}-\Delta \delta^{i}\left[E_{i j k l} \frac{\partial \bar{t}}{\partial t_{k l}}\right] \\
(\kappa)_{i+1}^{n+1} & =(\kappa)_{i}^{n+1}-\Delta \delta^{i} E_{p} \\
(t)_{i+1}^{n+1} & =(t)_{i}^{n+1}+\tau_{i} \log \left(\frac{F_{i}^{n+1}}{F_{i+1}^{n+1}}\right)
\end{aligned}
$$

The set of eqns (79) are used successively over a series of segments within the time step, to update the stresses and the yield surface till the value of the accumulated time $t_{i+1}$ exceeds the value of time step $\Delta t$. At this point, the parameter is redefined as:

$$
\Delta \delta=\gamma F_{i}^{n+1} \tau_{i}\left[1-e^{-\left(\Delta t-t_{i}\right) / \tau}\right]
$$

and the eqns (79) are solved for the final values of $t$ and $\kappa$. It can be seen that though the stress is made to relax over the exact time step $\Delta t$ in the above algorithm, the final stress state may not always lie on the updated yield surface because of the linearization in each segment. For an exponent of the power law different from 1, the equations have to be modified accordingly though the procedure will still remain the same.

\section{COMPUTATIONAL PROCEDURES}

Sheet metal forming is a time dependent process and thus, its finite element analysis is carried out in increments over a discrete number of time steps. Within each time step computation of the displacements, stresses and temperatures are carried out using the schemes that have been discussed in the preceeding sections. Owing to the implicit methods used in the temporal integration within the discretized weak forms, iterative solution of the resulting system of equations is indispensable. In the traditional Newton-Raphson method the stiffness matrix has to be computed at every iteration step. However, in this analysis, the nature of the constitutive relations and the added terms due to the ALE formulation makes this computation very expensive, particularly because of the evaluation of $\mathbf{R}$ at each integration point. This, and a number of other reasons that have been discussed in $[26,27]$ makes the quasi-Newton methods (e.g. the BFGS method) a more desirable alternative to the Newton-Raphson method of iteration for this class of problems. Within an outer loop of iteration for the overall stress and displacement convergence, iteration is performed for temperatue increments, stress relaxation due to temperature and viscoplasticity. Once the iteration has converged, it is necessary to update the path dependent variables to the grid points. Details of the solution procedure are not presented in this paper and will be discussed in a future paper. However a flow chart showing the overall scheme is given in Fig. 5.

\section{CONCLUSION}

The accurate simulation of the sheet metal forming problem requires careful considerations of several important aspects of mechanics. In this paper an attempt has been made to couple various observations into a detailed finite element analysis. The correctness of the simulation depends on the relative accuracy of each of the algorithms that have been implemented. Numerical results may be used to strengthen the validity of the method discussed here which will be presented in a forthcoming paper. 


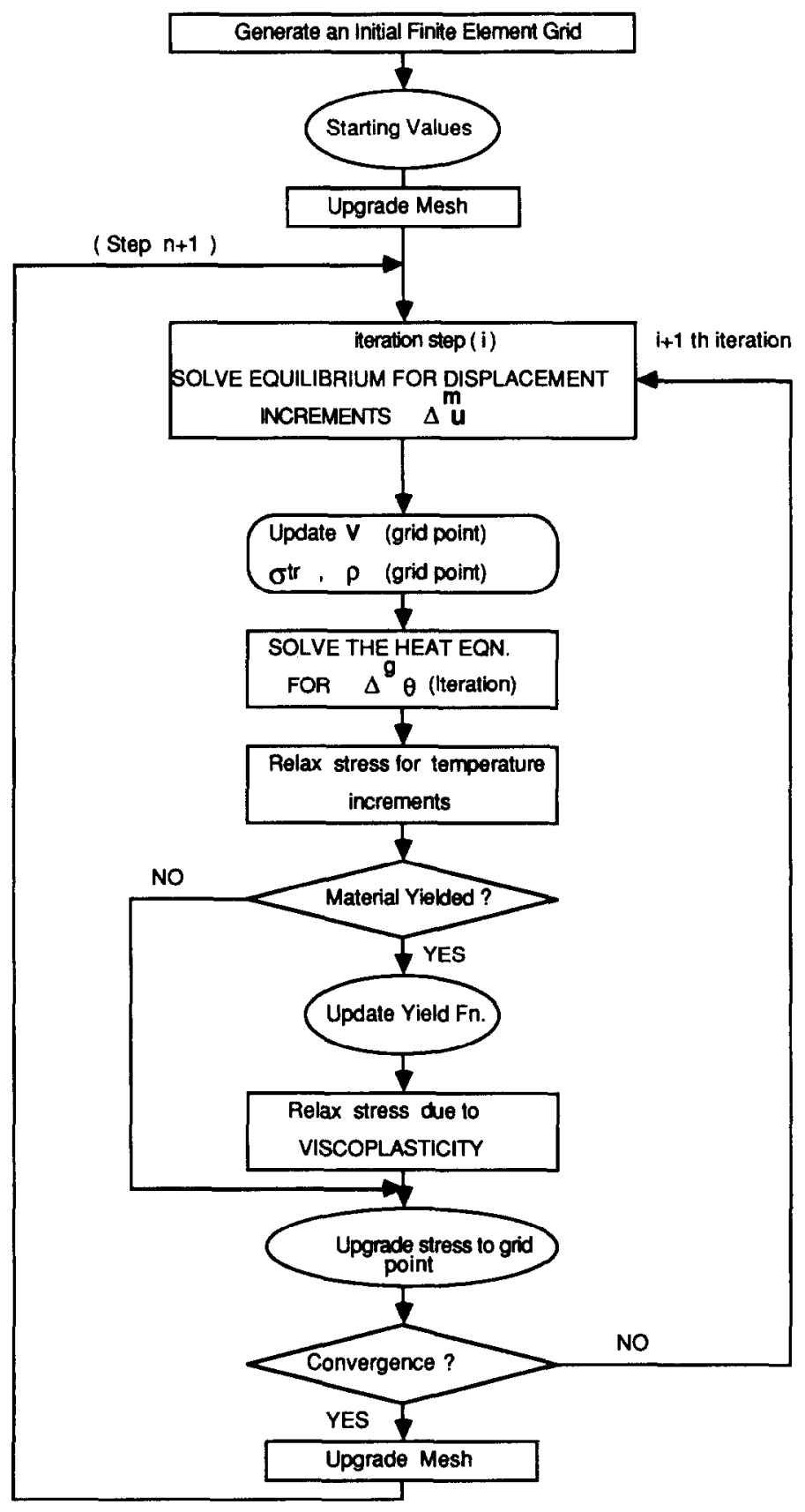

Fig. 5. Global solution algorithm.

\section{REFERENCES}

[1] C. W. HIRT, A. A. A. AMSDEN and J. L. COOK, All arbitrary Lagrangian-Eulerian computing method for all flow speed. J. Comput. Phys. 14, 227-253 (1974).

[2] J. DONEA, P. FASOLI-STELLA and S. GIULIANI, Lagrangian and Eulerian finite element techniques for transient fluid-structure interaction problems. Trans. 4th Int. Conf. on Smirt, Vol. B, pp. 1-12 (1977).

[3] J. M. KENNEDY and T. B. BELYTSCHKO, Theory and application of a finite element method for arbitrary Lagrangian-Eulerian and solids. Nucl. Engng Design 68, 129-146 (1981).

[4] T. R. HUGHES, W. K. LIU and T. ZIMMERMAN, Lagrangian-Eulerian finite element formulation for incompressible viscous flows, U.S.-Japan Seminar on interdisciplinary finite element analysis, Cornell University, Ithaca, New York (August 1979).

[5] R. B. HABER, A mixed Eulerian-Lagrangian displacement model for large deformation analysis in solid mechanics. Comput. Meth. Appl. Mech. Engng 43, 277-292 (1984).

[6] T. J. R. HUGHES and T. BELYTSCHKO, A precis of developments in computational methods for transient analysis. J. Appl. Mech. 50, 1033-1041 (1983). 
[7] O. C. ZIENKIEWICZ, Finite Elements in time-domain State of the Art Surveys on Finite Element Technology, (A. K. Noor and W. D. Pilkey, eds), ASME (1983).

[8] P. PERZYNA, Fundamental problems in viscoplasticity. Adv. Appl. Mech. 9, 243-377 (1966).

[9] P. M. PINSKY, K. S. PISTER and R. L. TAYLOR, Formulation and numerical integration of elastoplastic and elastic-viscoplastic rate constitutive equations. Mechanics of Engng. Matherials, (C. S. Desai and R. H. Gallagher, eds). Wilev (1984).

[10] J. C. NAGTEGAAL and J. E. DEJONG, Some aspects of nonisotropic work hardening in finite deformation plasticity. Proceedings of the workshop. Plasticity at finite deformation. Division of Applied Mechanics, Stanford, University, Stanford, Calif. (1981).

[11] G. C. JOHNSON and D. J. BAMMANN, A discussion of stress rates in finite deformation problems, Sandia Report, SAND 82-8821 (1982).

[12] A. E. GREEN and P. M. NAGHDI, A general theory of an elastic-plastic continuum. Arch. Ratl. Mech. Anal. 18, 251-281 (1965).

[13] J. C. NAGTEGAAL, On the implementation of inelastic constitutive equations with special reference to large deformation problems. Comput. Meth. Appl. Mech. Engng 33, 469-484 (1982).

[14] R. HILL, A theory of yielding and plastic flow of anisotropic metals. Proc. R. Soc. London, Ser. A, 193 (1948).

[15] L. W. HU, Studies on plastic flow of anisotropic metals. J. Appl. Mech., Sept. 1956, 444-450 (1956).

[16] M. L. WILKINS, Calculation of Elastic-Plastic flow methods of Computational Physics, Vol. 3 (B. Adler et al., eds) Academic Press, New York (1964).

[17] R. D. KREIG and S. W. KEY, Implementation of a time independent plasticity theory into structural computer programs. Constitutive Equations in Viscoplasticity: Computational and Engineering Aspects (J.A. Stricklin and $\mathrm{K}$. J. Saczalski, eds), AMD-20, ASME, New York.

[18] T. J. R. HUGHES, Numerical Implementation of Constitutive Models: Rate-independent Deviatoric Plasticity, Theoretical Foundations for Large Computations of Nonlinear Rehavior (S. Nemat-Nasser, R. J. Asaro and G. A. Hegemier, eds). Martinus Nijhoff Publishers (1984).

[19] S. KOBAYASHI, Thermoviscoplastic analysis of metal forming problems by the finite element method. Numerical Methods in Industrial Forming Processes (J. F. Pittman, R. D. Wood, J. M. Alexander and O. C. Zienkiewicz, eds), Pineridge Press, Swansea, U.K. (1982).

[20] P. R. DAWSON and E. G. THOMPSON, Finite element analysis of steady state elasto viscoplastic flow by the initial stress rate method. Int. J. Num. Meth. Engng 12, 47-57 (1978).

[21] L. M. TAYLOR and E. B. BECKER, Some computational aspects of large deformation, rate dependent plasticity problems. Comput. Meth. Appl. Mech. Engng 41, 251-277 (1983).

[22] N. M. WANG and B. BUDIANSKY, Analysis of sheet metal stamping by a finite element method. J. Appl. Mech. ASME 45, 73 (1978).

[23] N. M. WANG and M. L. WENNER, Elastic viscoplastic analysis of simple stretch forming problems. Mechanics of Sheet Metal Forming, (D. P. Koistinen and N. M. Wang, eds). Plenum Press, New York (1978).

[24] T. J. R. HUGHES, Unconditionally stable algorithms for nonlinear heat conduction. Comput. Meth. App. Mech. Engng 10, 135-139 (1977).

[25] T. J. R. HUGHES, Analysis of transient algorithms with particular reference to stability behavior. In Computational Methods for Transient Analysis, Vol. 1 (T. Belytschko and T. J. R. Hughes, eds). N-H (1983).

[26] K. J. BATHE and A. P. CIMENTO, Some practical procedures for the solution of nonlinear finite element equations. Comput. Meth. Appl. Mech. Engng 22, 59-85 (1980).

[27] K. J. BATHE, Finite Element Procedures in Engineering Analysis. Prentice Hall, 1982.

[28] A. E. GREEN and B. C. MCINNIS, Generalized hypo-clasticity. Proc. R. Soc. Edinburgh A 57, 220 (1967).

[29] J. C. SIMO and J. E. MARSDEN, On rotated stress tensor and the material version of Doyle-Ericksen formula. Arch. Rat. Mech. Anal. 86, 213-231 (1984).

[30] M. ORTIZ and J. C. SIMO, An analysis of a new class of integration algorithms for elasto-plastic constitutive relations. Int. J. Num. Meth. Engng 23, 353-366 (1986).

[31] M. ORTIZ, P. M. PINSKY and R. L. TAYLOR, Operator split method for the numerical solution of the elasto-plastic dynamic problem. Comput. Meth. Appl. Mech. Engng 39, 137-157 (1983).

[32] T. J. R. HUGHES and J. WINGET, Finite rotation effects in numerical integration of rate constitutive arising in large-deformation analysis. Int. J. Num. Meth. Engng 15, 1862-1867.

(Received 2 July 1987) 